\title{
ECNU at SemEval-2018 Task 2: Leverage Traditional NLP Features and Neural Networks Methods to Address Twitter Emoji Prediction Task
}

\author{
Xingwu Lu ${ }^{1}$, Xin Mao ${ }^{1}$, Man Lan ${ }^{1,2 *}$, Yuanbin $\mathbf{W u}^{1,2}$ \\ ${ }^{1}$ Department of Computer Science and Technology, \\ East China Normal University, Shanghai, P.R.China \\ ${ }^{2}$ Shanghai Key Laboratory of Multidimensional Information Processing \\ $\{51174506023,10131530334\}$ astu.ecnu.edu.cn \\ $\{$ mlan, ybwu\}ecs.ecnu.edu.cn
}

\begin{abstract}
This paper describes our submissions to Task 2 in SemEval 2018, i.e., Multilingual Emoji Prediction. We first investigate several traditional Natural Language Processing (NLP) features, and then design several deep learning models. For subtask 1: Emoji Prediction in English, we combine two different methods to represent tweet, i.e., supervised model using traditional features and deep learning model. For subtask 2: Emoji Prediction in Spanish, we only use deep learning model.
\end{abstract}

\section{Introduction}

Visual icons play a crucial role in providing information about the extra level of social media information. SemEval 2018 shared task for researcher$\mathrm{s}$ to predict, given a tweet in English or Spanish, its most likely associated emoji (Barbieri et al., 2018, 2017) (Task 2, Multilingual Emoji Prediction), which is organized into two optional subtask (subtask 1 and subtask 2) respectively in English and Spanish.

For subtask 1, we adopt a combination model to predict emojis, which consists of traditional Natural Language Processing (NLP) methods and deep learning methods. The results returned by the classifier with traditional NLP features, by the neural network model and by the combination model are voted to get the final result. For subtask 2, we only use deep learning model.

\section{System Description}

For subtask 1, we explore three different methods i.e., using traditional NLP features to learn a supervised machine learning-based classifier, learning a deep learning model to make prediction and combine features captured by neural networks with traditional NLP features to train a supervised machine learning-based classifier. For subtask 2, we simply implement deep learning method to make prediction.

\subsection{Traditional NLP Features}

In this task, we extract the following three types of features to capture effective information from the given tweets, i.e., linguistic features, sentiment lexicon features and tweet specific features.

\subsubsection{Linguistic Features}

- N-grams: We extract 3 types of Bag-ofWords features as $\mathrm{N}$-grams features, where $\mathrm{N}$ $=1,2,3$ (i.e., unigram, bigram, and trigram features).

- POS: Generally, the sentences carrying subjective emotions are inclined to contain more adjectives and adverbs while the sentences without sentiment orientation would contain more nouns. Thus, we extract POS tag from the sentence as features with the Bag-ofWords form.

- Correlation Degree: For each word appear in training data, the ratio of the number of occurrences under each class and the total occurrences is counted as the correlation degree of the word to a certain class. When the feature is created, the sum of the correlation degree of words in tweet is counted as the correlation degree of the tweet to a certain class:

$$
\operatorname{CorrDeg}(s, l)=\sum_{t=1}^{|s|} \frac{O\left(w_{t}, c_{l}\right)}{\sum_{i}^{N} O\left(w_{t}, c_{i}\right)}
$$

Where $|s|$ is the length of tweet and $N$ is the number of classes, $w_{t}$ means $t^{t h}$ word in tweet and $c_{i}$ means $i^{t h}$ class, $O\left(w_{t}, c_{i}\right)$ denotes the number of tweets of $c_{i}$ that contain $w_{t}$. The dimension of this feature is equal to the number of classes, value is correlation 
degree of the tweet to each class, i.e., Cor$r \operatorname{Deg}(s, l)$.

\subsubsection{Sentiment Lexicon Features (SentiLexi)}

We also extract sentiment lexicon features (SentiLexi) to capture the sentiment information of the given sentence. Given a tweet, we first convert al1 words into lowercase. Then on each sentiment lexicon, we calculate the following six scores for one message: (1) the ratio of positive words to all words, (2) the ratio of negative words to all word$\mathrm{s}$, (3) the maximum sentiment score, (4) the minimum sentiment score, (5) the sum of sentiment scores, (6) the sentiment score of the last word in tweet. If the word does not exist in one sentiment lexicon, its corresponding score is set to 0 . The following 8 sentiment lexicons are adopted in our systems: Bing Liu lexicon ${ }^{1}$, General Inquirer lexicon $^{2}, I M D B^{3}, M P Q A^{4}, N R C$ Emotion Sentiment Lexicon $^{5}$, AFINN $^{6}$, NRC Hashtag Sentiment Lexi$\mathrm{con}^{7}$, and NRC Sentiment 140 Lexicon $^{8}$.

\subsubsection{Tweet Specific Features}

- Punctuation: Considering that users often use exclamation marks and question marks to express strongly surprised and questioned feelings, we extract 7-dimensions punctuation features by recording rules of punctuation marks in the tweets.

- All-caps: One binary feature is to check whether this tweet has words in uppercase.

- Bag-of-Hashtags: We construct a vocabulary of hashtags appearing in the training data and then adopt the bag-of-hashtags method for each tweet.

\subsection{Deep Learning Modules}

In addition to manually constructing features, we build deep neural models to capture the semantics of the text. Figure 1 shows the network structure of our model. The input of the network is a tweet, which is a sequence of words. The output of the network contains class elements.

\footnotetext{
${ }^{1}$ http://www.cs.uic.edu/liub/FBS/sentiment-analysis.html

${ }^{2} \mathrm{http} / / / \mathrm{www} . w j h . h a r v a r d . e d u /$ inquirer/homecat.htm

${ }^{3} \mathrm{http}: / /$ www.aclweb.org/anthology/S13-2067

${ }^{4} \mathrm{http}: / /$ mpqa.cs.pitt.edu/

${ }^{5}$ http://www.saifmohammad.com/WebPages/lexicons.html

${ }^{6} \mathrm{http}: / / \mathrm{www} 2 . i m m . d t u . d k / p u b d b / v i e w s /$ publication details.php?id=6010

${ }^{7}$ http://www.umiacs.umd.edu/saif/WebDocs/NRC-

Hashtag-Sentiment-Lexicon-v0.1.zip

${ }^{8} \mathrm{http} / / /$ help.sentiment140.com/for-students/
}

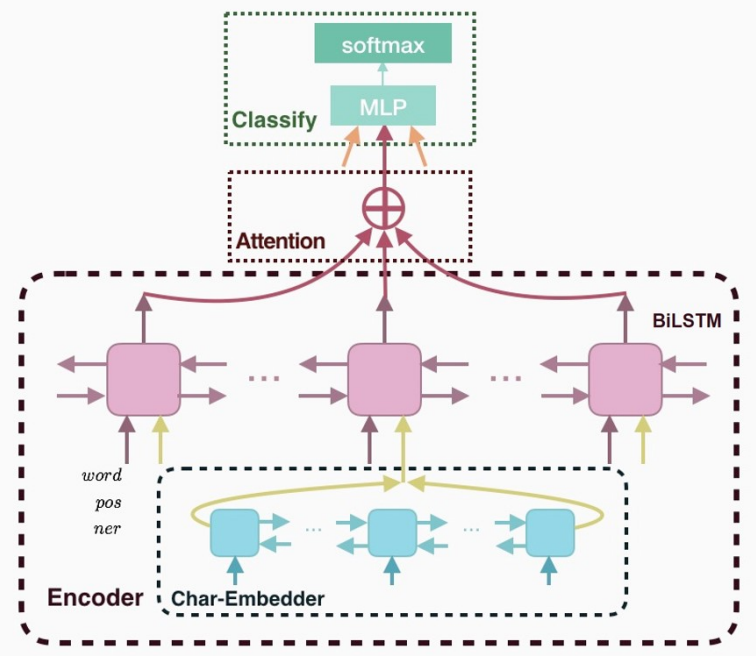

Figure 1: Deep learning model architecture.

\subsubsection{Word-Level Representations}

We use pre-trained word embedding concatenated with char embedding, POS embedding and $\mathrm{N}$ ER embedding obtaining a final representation for each word type, which are learned together with the updates to the model.

- Word Embedding: Word embedding is a continuous-valued vector representation for each word, which can capture meaningful syntactic and semantic regularities. In this task, we use the 300-dimensional word vectors pre-trained on Twitter provided by SemEval task organizers, available in $S W M^{9}$

- Char Embedding: We randomly initialize the representation of the character and compute character-based continuous-space vector embeddings of the words in tweets by bidirectional LSTM. The dimension of char embedding is 50 .

- POS Embedding: We randomly initialize the representation of the POS tag in tweet with a vector size of 50 .

- NER Embedding: We also randomly initialize the representation of the NER tag in tweet with a vector size of 50 .

\subsubsection{Sentence-Level Representations}

- Bi-Directional LSTM: We apply a recurrent structure to capture contextual information as far as possible when learning word representations, to model the tweet with both of the

\footnotetext{
${ }^{9}$ https://github.com/fvancesco/acmmm2016
} 
preceeding and following contexts, we apply a Bi-directional Long Short-term Memory Networks (BiLSTM, Graves et al. (2005)) architecture as shown in Figure 1.

- Attention Mechanism: Considering not al1 words contribute equally to the representation of the sentence meaning, we introduce attention mechanism (Bahdanau et al., 2014) to extract such words that are important to the meaning of the sentence and aggregate the representation of those informative words to form a sentence vector.

We first use BiLSTM and Attention Mechanism to obtain sentence-level representations and then concatenate it with several effective NLP features. At last, we use a Multi-layer Perceptron (MLP) and output the probability of emoji label based on a softmax function. The BiLSTM has a hidden size of 512. The MLP have 1 hidden layer of size 200 and relu non-linearity.

To learn model parameters, we minimize the KL-divergence between the outputs and gold labels. We adopt Adam (Kingma and Ba, 2014) as optimization method and set learning rate of 0.01 .

\section{Experimental Settings}

\subsection{Datasets}

For training sets, the organizers provide only the list of tweet ID and a script for all participants to collect tweets. However, since not all tweet$\mathrm{s}$ are available when downloading, participants may collect slightly different numbers of tweet$\mathrm{s}$ for training sets. In addition, we find that the crawled training sets and the trial sets provided by the organizers have $37.26 \%$ overlap in English and $71.16 \%$ in Spanish. So we remove the duplicate data and combine train and trial sets to perform a 3 -fold cross-validation. Table 1 shows the statistics of the tweets we collect in our experiments. In subtask 1 , the number of class 0 is the largest, accounting for $22.28 \%$, followed by class 1 and class 2 , respectively, $10.37 \%$ and $10.20 \%$, and the other 17 classes distribute between $2.46 \%$ and $5.51 \%$. Subtask 2 has a similar data distribution.

\subsection{Data Preprocessing}

Firstly, we convert unicode encoding into corresponding characters, punctuation, emoticons. Then we use slangs ${ }^{10}$ to transform the informal

\footnotetext{
${ }^{10}$ https://github.com/haierlord/resource/blob/master/slangs
}

\begin{tabular}{|c|c|c|}
\hline label Dataset & Subtask 1 & Subtask 2 \\
\hline 0 & $116693(22.28 \%)$ & $20495(20.41 \%)$ \\
\hline 1 & $54313(10.37 \%)$ & $13688(13.63 \%)$ \\
\hline 2 & $53432(10.20 \%)$ & $9342(9.30 \%)$ \\
\hline 3 & $28855(5.51 \%)$ & $6859(6.83 \%)$ \\
\hline 4 & $25778(4.92 \%)$ & $6535(6.51 \%)$ \\
\hline 5 & $24475(4.67 \%)$ & $4765(4.43 \%)$ \\
\hline 6 & $22301(4.26 \%)$ & $4444(4.42 \%)$ \\
\hline 7 & $19236(3.67 \%)$ & $3868(3.85 \%)$ \\
\hline 8 & $17908(3.42 \%)$ & $3687(3.67 \%)$ \\
\hline 9 & $16996(3.25 \%)$ & $3544(3.53 \%)$ \\
\hline 10 & $16755(3.20 \%)$ & $3399(3.16 \%)$ \\
\hline 11 & $16052(3.07 \%)$ & $2943(2.93 \%)$ \\
\hline 12 & $15167(2.90 \%)$ & $2826(2.81 \%)$ \\
\hline 13 & $13650(2.61 \%)$ & $2727(2.72 \%)$ \\
\hline 14 & $14136(2.70 \%)$ & $2629(2.62 \%)$ \\
\hline 15 & $13963(2.67 \%)$ & $2564(2.55 \%)$ \\
\hline 16 & $13702(2.62 \%)$ & $2618(2.61 \%)$ \\
\hline 17 & $13474(2.57 \%)$ & $2551(2.54 \%)$ \\
\hline 18 & $13867(2.65 \%)$ & $2552(2.54 \%)$ \\
\hline 19 & $12900(2.46 \%)$ & - \\
\hline total & 523653 & 100440 \\
\hline
\end{tabular}

Table 1: The statistics of data sets in combination of training sets and trial sets which we used to perform a 3-fold cross-validation. The numbers in brackets are the percentages of different classes in each data set.

writing to regular forms, e.g., " $L O L$ " replaced by "laugh out loud". And we recover the elongated words to their original forms, e.g., "soooooo" to "so". Finally, we implement tokenization, POS tagging, named entity recognizing(NER) with the aid of Stanford CoreNLP tools (Manning et al., 2014).

\subsection{Learning Algorithm}

Considering the large dimension of the features designed by traditional NLP methods, we use learning algorithms of Logistic Regression(LR) to build classification models, which is supplied in Liblinear ${ }^{11}$.

\subsection{Evaluation Metrics}

The official evaluation measure is Macro F-score, which would inherently mean a better sensitivity to the use of emojis in general, rather than for instance overfitting a model to do well in the three or four most common emojis of the test data. Macro F-score can be defined as simply the average of the individual label-wise F-scores.

\footnotetext{
${ }^{11}$ https://www.csie.ntu.edu.tw/ cjlin/liblinear/
} 


\section{Experiments on Training Data}

\subsection{Comparison of NLP Features}

Table 2 lists the comparison of different contributions made by different features on crossvalidation with Logistic Regression algorithm. From the results in Table 2, we observe the following findings:

(1) The combination of Uigram, Bigram, Correlation Degree, POS and SentiLexi achieves the best performance (i.e., 34.63).

(2) Correlation Degree feature makes more contributes than other features, as it reflects the degree of relevance between tweets and emoji label.

(3) Bigram feature makes contribution and is more effective than unigram feature. The reason may be that bigram feature can capture more contextual information and word orders.

(4) SentiLexi feature also makes contribution, which indicates that SentiLexi features are beneficial not only in traditional sentiment analysis tasks, but also in predicting the emoji in tweet.

\begin{tabular}{c|c|c}
\hline Features & $\mathbf{F}_{\text {macro }}$ & change \\
\hline Best Features & 34.63 & - \\
\hline -Correlation Degree & 30.59 & -4.04 \\
-Bigram & 33.38 & -1.25 \\
-SentiLexi & 33.63 & -1.00 \\
-POS & 33.91 & -0.72 \\
-Uigram & 34.22 & -0.41 \\
\hline
\end{tabular}

Table 2: Performance of different features on subtask 1. - means to exclude some features.

\subsection{Comparison of Deep Learning Modules}

Table 3 shows the results of different deep learning models described before. From Table 3, we observe the findings as follows:

(1) We explore the performance of three different deep learning model: Neural Bag-ofWords(NBOW, Iyyer et al. (2015)), Convolutional Neural Network (CNN, Collobert et al. (2011)) and Bi-directional Long Short-term Memory Networks (LSTM, Hochreiter and Schmidhuber (1997)). All models used only pre-trained word embedding to compare. Clearly, BiLSTM outperformed other models in this task, and our deep learning model is based on BiLSTM.

(2) POS embedding makes more contribution than other word-level representations. Since POS embedding can learn emotional tendencies, it is beneficial for tweet emojis prediction.
(3) The last two rows results shows that combine both SentiLexi and Punctuation features with sentence representations to train the deep learning model can make contribution.

\begin{tabular}{c|c|c}
\hline Models & Subtask 1 & Subtask 2 \\
\hline NBOW & 23.73 & 18.67 \\
CNN & 23.64 & 18.91 \\
BiLSTM & 25.64 & 19.32 \\
\hline .+Char & $25.66(+0.02)$ & 19.56 \\
.+NER & $26.57(+0.91)$ & - \\
.+POS & $30.55(+3.98)$ & - \\
\hline .+Attention & $30.74(+0.19)$ & - \\
\hline .Punctuation & $32.10(+1.36)$ & - \\
.+SentiLexi & $32.59(+0.49)$ & - \\
\hline
\end{tabular}

Table 3: Performance of different deep learning models on subtask 1 and subtask 2. .+ means to add current module to the previous model. The numbers in the brackets are the performance increments compared with the previous results.

\subsection{Combination and Ensemble}

For subtask 1, we also use the trained neural networks described in 4.2 to capture the features of tweets and combine it with traditional NLP features to train a Logistic Regression classifier, named Combination Model.

Table 4 shows the results of different methods. We find that combination model improved the performance and the ensemble of 3 methods achieve the best result. It suggests that the traditional NLP methods and the deep learning models are complementary to each other and their combination achieves the best performance.

\begin{tabular}{l|l}
\hline Methods & F $_{\text {macro }}$ \\
\hline Traditional NLP Features & 34.63 \\
\hline Deep Learning Model & 32.59 \\
\hline Combination Model & 35.21 \\
\hline Ensemble Model & 35.57 \\
\hline
\end{tabular}

Table 4: Performance of different methods on subtask 1.

\subsection{System Configuration}

Based on above experimental analysis, the two system configurations on test data sets are listed as followings:

(1) subtask 1: Logistic Regression with best NLP feature sets is used as model 1. Deep learn- 
ing model is used as model 2. Logistic Regression with NLP features and the feature captured by deep learning model is used as model 3. Ensemble of three models is used as final submission.

(2) subtask 2: Deep learning model with word embedding and char embedding is used as submission.

\section{Results on Test Data}

\begin{tabular}{c|c|c}
\hline & Subtask 1 & Subtask 2 \\
\hline Our system & $33.35(5)$ & $16.41(7)$ \\
\hline Rank 1 & $35.99(1)$ & $22.36(1)$ \\
Rank 2 & $35.36(2)$ & $18.73(2)$ \\
Rank 3 & $34.02(3)$ & $18.18(3)$ \\
\hline
\end{tabular}

Table 5: Performance of our systems and the topranked systems for two subtasks on test datasets. The numbers in the brackets are the official rankings.

Table 5 shows the results on test datasets. From Table 5, we find that our system achieves almost the same performance as the cross-validation. The low performance of this task illustrates the difficulty of the task itself, especially the Spanish task.

\section{Conclusion}

In this paper, we extract several effective traditional NLP features, design different deep learning models and build a model in combination of traditional NLP features and deep learning method together. The extensive experimental results show that this combination improves the performance.

For the future work, we consider to focus on developing a neural networks model to handle unbalanced data and improve the performance of confusing labels.

\section{Acknowledgements}

This work is is supported by the Science and Technology Commission of Shanghai Municipality Grant (No. 15ZR1410700) and the open project of Shanghai Key Laboratory of Trustworthy Computing (No.07dz22304201604).

\section{References}

Dzmitry Bahdanau, Kyunghyun Cho, and Yoshua Bengio. 2014. Neural machine translation by jointly learning to align and translate. arXiv preprint $\operatorname{arX}$ iv: 1409.0473 .

Francesco Barbieri, Miguel Ballesteros, and Horacio Saggion. 2017. Are emojis predictable? In Proceedings of the 15th Conference of the European
Chapter of the Association for Computational Linguistics: Volume 2, Short Papers, pages 105-111. Association for Computational Linguistics.

Francesco Barbieri, Jose Camacho-Collados, Francesco Ronzano, Luis Espinosa-Anke, Miguel Ballesteros, Valerio Basile, Viviana Patti, and Horacio Saggion. 2018. SemEval-2018 Task 2: Multilingual Emoji Prediction. In Proceedings of the 12th International Workshop on Semantic Evaluation (SemEval-2018), New Orleans, LA, United States. Association for Computational Linguistics.

Ronan Collobert, Jason Weston, Léon Bottou, Michael Karlen, Koray Kavukcuoglu, and Pavel Kuksa. 2011. Natural language processing (almost) from scratch. Journal of Machine Learning Research, 12(Aug):2493-2537.

Alex Graves, Santiago Fernández, and Jürgen Schmidhuber. 2005. Bidirectional lstm networks for improved phoneme classification and recognition. In International Conference on Artificial Neural Networks, pages 799-804. Springer.

Sepp Hochreiter and Jürgen Schmidhuber. 1997. Long short-term memory. Neural computation, 9(8):1735-1780.

Mohit Iyyer, Varun Manjunatha, Jordan Boyd-Graber, and Hal Daumé III. 2015. Deep unordered composition rivals syntactic methods for text classification. In Proceedings of ACL 2015.

Diederik P Kingma and Jimmy Ba. 2014. Adam: A method for stochastic optimization. arXiv preprint arXiv:1412.6980.

Christopher D. Manning, Mihai Surdeanu, John Bauer, Jenny Finkel, Steven J. Bethard, and David McClosky. 2014. The Stanford CoreNLP natural language processing toolkit. In Proceedings of 52nd Annual Meeting of the Association for Computational Linguistics: System Demonstrations, pages 5560 